\title{
HOW ELECTRICAL AND CHEMICAL REQUIREMENTS FOR REFETS MAY COINCIDE
}

\author{
P. BERGVELD, A. VAN DEN BERG, P. D. VAN DER WAL, \\ M. SKOWRONSKA-PTASINSKA, E. J. R. SUDHÖLTER and D. N. REINHOUDT
}

Department of Electrical Engineering and Laboratory of Organic Chemistry, University of Twente, P.O. Box 217, 7500 AE Enschede (The Netherlands)

(Received April 21, 1988; in revised form October 7, 1988; accepted November 11, 1988)

\begin{abstract}
After discussing the features of a differential ISFET/REFET measuring concept, the published attempts to construct a proper REFET are summarized. It is concluded that the present REFETs are based upon the addition of a blocking polymeric layer to the gate surface of an ISFET, but that this approach fails with respect to the required insensitivity to ionic strength variations as well as with respect to the electrical stability.

As a solution to these problems, this paper describes the development of a REFET concept that is based on the chemical attachment of a nonblocking polymeric layer. Characterization methods of these layers with respect to the electrical as well as the chemical behaviour are given and discussed.

Finally, the experimental results of an acrylate/polyHEMA-REFET are shown in a differential ISFET/REFET system.
\end{abstract}

\section{Introduction}

Many papers have been published in the last decades concerning solidstate electrodes, especially ISFETs [1], but it is amazing that so little attention has been paid to the reference electrode, which inevitably has to be used in conjunction with these electrodes. Although some attempts have been made to copy the classical reference electrode in a silicon-based construction [2], the results are still not very promising with respect to the real application of these devices. The main reason is that the conceptual base of these reference electrodes is the use of an $\mathrm{Ag} / \mathrm{AgCl}$ electrode, which makes contact with the analyte by means of a liquid salt bridge and a porous plug. The smaller these devices are made, the shorter their lifetime due to the limited volume of internal electrolyte, which changes concentration relatively fast due to diffusion of different types of ions. Decreasing the diffusion rate by 
reducing the porosity of the plug results in an undesirable high-ohmic contact with the solution.

With respect to the development of a solid-state reference electrode, which has to be used in combination with an ISFET-type of ion-sensitive electrode, it is obvious that a reference electrode made by a technique compatible with FET technology would be preferable. In view of the problems mentioned above, it is also obvious that this reference electrode should not contain an internal electrolyte with a porous plug construction. The solution has been given by Matsuo, who introduced the concept of a REFET, which is essentially an ISFET whose gate surface is modified in such a way that it behaves as if chemically inert [3]. If it is possible to produce a chemically-stable REFET with identical electrical properties to those of an ISFET and the two can be integrated in one chip, a simple onchip evaporated noble metal can act as a pseudo- or quasi-reference electrode (QRE), to ground the analyte. Such a differential system is shown schematically in Fig. 1.

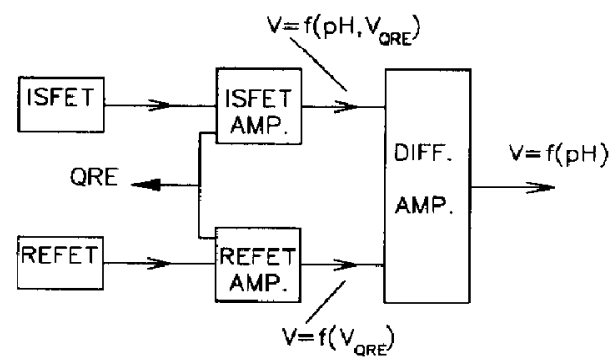

Fig. 1. Schematic representation of ISFET/REFET differential measuring system with quasi-reference electrode (QRE), e.g., Pt electrode.

Although the platinum-electrolyte interface is thermodynamically undefined, and thus generates an unstable voltage, this voltage is measured by the differential ISFET/REFET pair as a common mode voltage and will therefore not result in an amplifier output voltage. A change in the $\mathrm{pH}$ of the analyte will, however, be measured as a differential voltage, because the ISFET responds to the $\mathrm{pH}$ and the REFET does not.

The question is, whether it is possible to modify the gate surface of an ISFET in such a way that the resulting device will indeed not respond to pH nor to any other ion or change in concentration. Meanwhile, the original ISFET electrical characteristics should be retained, as they are necessary to keep a maximum of suppression of the common mode voltage variations originating from the QRE.

In the next Sections it will be shown how the mutual requirements for electrical and chemical REFET characteristics can be satisfied by an optimal choice of the composites of a particular membrane, covering the gate surface of an ISFET and deposited and attached in an ISFET-compatible technology. 


\section{Historical overview}

\section{The parylene REFET}

Based on the REFET concept mentioned in the Introduction, Matsuo described the first actual REFET made by modifying the gate of a $\mathrm{Si}_{3} \mathrm{~N}_{4}$ ISFET with a coating of parylene [3]. A parylene film deposited in a vacuum by thermal decomposition of di-p-xylylene has several promising characteristics, such as: (i) precise control of film thickness as well as uniformity; (ii) relatively pinhole-free films as thin as $100 \mathrm{~nm}$; (iii) excellent dielectric characteristics, and (iv) surface groups that can easily be chemically modified if desired. The thin ion-blocking layer [4] with a thickness of the order of the original inorganic oxide layer of an ISFET is especially important, because it does not influence the electrical sensitivity of the ISFET drastically, a necessary requirement with respect to the common mode rejection ratio of the differential ISFET/REFET amplifier. It appeared, unfortunately, that Matsuo's original parylene coatings failed with respect to the suppression of the $\mathrm{pH}$ sensitivity, which was sometimes 10 $30 \mathrm{mV}$ per decade $\mathrm{pH}$ from $\mathrm{pH} 1$ to 13 . Careful analysis of cleaning procedures and different chemical treatments of $\mathrm{Si}_{3} \mathbf{N}_{4}$ preceding the parylene deposition, as well as various deposition conditions and chemical treatments of the deposited layers, all carried out by Fujihira et al. [5] showed, however, that a decrease of $\mathrm{pH}$ sensitivity to as low as $4 \mathrm{mV}$ per decade $\mathrm{pH}$ and even lower beyond $\mathrm{pH} 10$ and below $\mathrm{pH} 4$ is possible. This drastic decrease of $\mathrm{pH}$ sensitivity might seem to be enough if one compares it with the $55 \mathrm{mV} / \mathrm{pH}$ of the corresponding ISFET of an ISFET/REFET pair. However, if the REFET response is subject to instabilities due to contamination or propagating chemical reactions, for example, the resulting change in sensitivity directly determines the overall accuracy of the ISFET/ REFET pair. A change of $0.5 \mathrm{mV}$ in the REFET response will already cause a deviation of $0.01 \mathrm{pH}$. Fujihira et al. [5] do not describe the stability of their parylene REFET, nor the actual source of the small pH sensitivity obtained. As long as the operational chemistry at the parylene/electrolyte interface is not clear, nothing can be said concerning the stability of this type of REFET. However, in Section 3 of this paper a serious drawback will be described concerning the intrinsic sensitivity to the electrolyte concentration, which phenomenon can be considered as a quasi-instability.

\section{Chemical surface modification}

The question arises whether it is possible to decrease the $\mathrm{pH}$ sensitivity of an inorganic gate surface, such as $\mathrm{SiO}_{2}$ or $\mathrm{Al}_{2} \mathrm{O}_{3}$, below the required level by capturing chemically the surface hydroxyl groups that ultimately determine the $\mathrm{pH}$ response. According to the site-dissociation model [6], the number of surface sites is represented by $N_{\mathrm{s}}$. With a decreased number of sites, say $N_{\mathrm{s}}{ }^{\prime}$, the maximum surface charge in the acidic or basic region is $q N_{\mathrm{s}}^{\prime}$, assuming that in these extreme cases all sites have reacted. This charge loads the double-layer capacitance. At sufficiently high concentrations 
$(C>0.1 \mathrm{M})$ the double-layer capacitance is determined by the capacity of the Helmholtz layer, for which a value of $20 \mu \mathrm{F} / \mathrm{cm}^{2}$ is usually taken. If over the full $\mathrm{pH}$ range a voltage from $+0.25 \mathrm{mV}$ to $-0.25 \mathrm{mV}$ is allowed, it means that the double layer is loaded with a charge of $10^{-8} \mathrm{C} / \mathrm{cm}^{2}$, the value of $q N_{\mathrm{s}}^{\prime}$ mentioned above. With $q$ as the elementary charge of $1.6 \times$ $10^{-19} \mathrm{C}$, this means that the decreased number of surface sites $N_{s}^{\prime}$ should be $6 \times 10^{10} / \mathrm{cm}^{2}$. In practice, the upper limit of $N_{\mathrm{s}}$ in oxides lies around $10^{15} /$ $\mathrm{cm}^{2}$, which means that a decrease to $0.005 \%$ should be realized in order to create a surface that responds only to a pH change of 0.01 .

Van den Berg et al. [7] showed that such a drastic reduction cannot be reached by a heat treatment followed by modification of the surface with various silylating agents, such as trichlorovinylsilane, trimethylchlorosilane, hexamethyldisilazane, 3-aminopropyl-triethoxy silane and butyldimethylsilylchloride. The conclusion of these investigations was that even a careful silylating procedure will never capture enough $\mathrm{OH}$ sites to eliminate pH sensitivity completely.

A solution to this problem could be the attachment of another material to chemically-reactive groups of the silylating agent, resulting in a certain bridging over the unreacted sites of the oxide surface with a thin film of at least several molecular layers. It appears, however, that in that case the $\mathrm{pH}$ sensitivity still hardly decreases, as was also shown by van den Berg et al. [7], who attached to a silylated $\mathrm{Ta}_{2} \mathrm{O}_{5}$ surface a polystyrene layer by a thermally-initiated radical polymerization of styrene. The ISFET with a layer of 5-10 nm polystyrene still showed a linear response to $\mathrm{pH}$ with a hardly decreased sensitivity, most probably due to pinholes in such a thin film. It cannot be excluded that the parylene REFETs described in the previous Section also suffer from this problem.

The conclusion of the results mentioned above is that very thin layers of polymeric materials can probably not easily be made pinhole free. This might be why in the literature concerning the development of REFETs thicker insulating layers, i.e., ion-blocking layers [4] are also applied, up to the micron level.

\section{Application of relatively thick blocking layers}

Nakajima et al. [8] reported the results of a REFET that was covered with a $200 \mathrm{~nm}$ Teflon membrane, deposited on a $\mathrm{Si}_{3} \mathrm{~N}_{4}$ ISFET by ion-beam sputtering. Teflon was chosen because of its well-known hydrophobicity, which was expected to be a criterion for $\mathrm{pH}$ insensitivity. Unfortunately, the devices show only some $\mathrm{pH}$ insensitivity in the range $\mathrm{pH} 4$ to $\mathrm{pH} 7$. It was suggested that the remaining $\mathrm{pH}$ sensitivity results from difluoroacetic acid derivatives on the surface of the Teflon, which indicates that hydrophobicity is not the only criterion for inertness of a material.

Tahara et al. developed a REFET that was coated with a plasmapolymerized styrene film with a thickness of $0.4 \mu \mathrm{m}$ [9]. The REFET remains stable against $\mathrm{pH}$ variations in the range $\mathrm{pH} 2-12$, but exact data are not given due to stability problems of the measuring set-up, a phenome- 
non that can most probably be ascribed to the low electrical sensitivity of the constructed REFET, as will be outlined in the next Section.

Van den Berg et al. tried to develop a REFET by coating an $\mathrm{SiO}_{2}$ ISFET with a photopolymerized layer of Bis-acryl [10] (see the following Section for preparation). It was shown by means of impedance measurements that the layer, having a thickness of $0.4-1.0 \mu \mathrm{m}$, behaves like an insulator and is indeed ion-blocking. This result was confirmed with SIMS investigations. Cationic ion profiles recorded before and after immersion of the polymer in an aqueous $10^{-3} \mathrm{M} \mathrm{KCl}$ solution are given in Figs. 2(a) and (b).

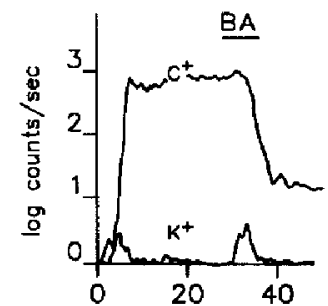

(a) sputt. time (min.)

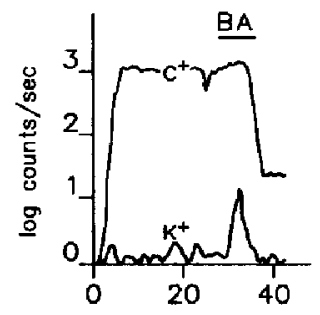

(b) sputt. time (min.)

Fig. 2. Cationic SIMS profiles of a photopolymerized layer of Bis-acryl, (a) before and (b) after immersion in aqueous $10^{-3} \mathrm{M} \mathrm{KCl}$ for $2 \mathrm{~h}$.

From the SIMS profile it is concluded that no substantial uptake of ions took place, because no significant potassium signal was measured, except at a sputtering depth corresponding to the polymer $/ \mathrm{SiO}_{2}$ interface. This signal might be attributed to surface contaminants. Unfortunately Bis-acryl shows a remaining $\mathrm{pH}$ sensitivity, probably resulting from residual acrylic acid functionalities in the film.

From the experimental results described in this and the previous Sections, it may be concluded that the application of thin as well as thick ion-blocking layers on top of the gate oxide of an ISFET has not yet resulted in a reliable REFET. The first reason is that really inert materials that have a sufficiently low concentration of sites are probably rare. The second reason is that pinhole-free layers, such as Bis-acryl or Teflon, should have a thickness of the order of at least some tenths of a micron, which may decrease the electrical sensitivity of the REFET so far that stable operation can no longer be guaranteed, as observed by Tahara $e t a l$. [9]. This effect will be considered in the next Section.

\section{Electrical problems with REFETs}

In the previous Sections most attention has been paid to the chemical characterization of REFETs. As mentioned in the Introduction, REFETs should be operated in a differential measuring set-up with the corresponding ISFET, in order to be able to use a quasi-reference electrode, preferably 


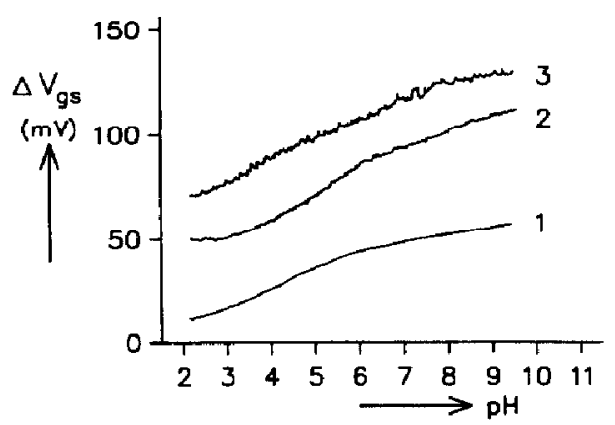

Fig. 3. Registration of REFET response with Bis-acryl layers of increasing thickness (curves 1 to 3 ).

consisting of a layer of a noble metal evaporated on the ISFET/REFET chip. Such a system is better the more equal the electrical sensitivities of the two devices are, because the voltage generated by the noble metal should be cancelled as a common mode voltage, which means that both devices should react in the same way to this voltage. However, covering an ISFET with a rather thick insulating layer drastically increases the effective overall thickness of the gate dielectric. Assuming the same dielectric constants for the two layers, this sandwiching decreases the gate capacity proportionally, as is also the case with the REFET electrical transconductance. This decrease in transconductance can be so drastic that even the biasing of the device under constant drain current conditions, as is usually applied, is no longer self evident. This is demonstrated in Fig. 3, where the output voltage is shown of a source-drain follower ISFET amplifier, to which a Bis-acryl coated ISFET was connected. Apart from the fact that the application of Bis-acryl does not suppress the $\mathrm{pH}$-sensitivity sufficiently, as outlined in the previous Section, the curves of Fig. 3 illustrate clearly the measuring problems of the device biasing.

The 'noisy' voltage is the result of the unstable biasing of the device, although the amplifier is feedback controlled with an open-loop amplification of 10000 . The instability problem increases with the thickness of the additional layer (curves 1 to 3 ). The top-top value of the 'noise' of $10 \mathrm{mV}$ is, of course, much too high to use the REFET as a reference device. With a Bis-acryl layer of approximately $1.5 \mu \mathrm{m}$, the transconductance of the original ISFET decreases from $600 \mu \mathrm{A} / \mathrm{V}$ to $35 \mu \mathrm{A} / \mathrm{V}$ (curve 3 ).

Another drawback of the REFET concept with an ion-blocking top layer of any thickness of a chemically inert material is the influence of the charge of the double-layer capacitance, which is dependent on the ionic strength. This phenomenon has already been described by Nakajima et al. [8] and further extensively analysed by Matsuo et al. [11]. Although an inert material does not set up an interfacial potential at the liquid interface, in practice the REFET will bias itself with a certain gate-source voltage, due to the operation in the constant drain-current mode. This voltage across the sandwiched gate dielectric results in a diffuse charge in the liquid at the 
polymer-liquid interface, with corresponding voltage drop. The value of this voltage is a function of the ionic strength, and therefore influences the device response negatively. The solution to this problem is also given by Matsuo et al. [11], namely biasing the REFET in such a way that the voltage over the total gate dielectric is zero. Matsuo proposes to meet this condition by applying a compensating substrate-source voltage, but any other electrical trimming can do the job. This virtually eliminates the influence of the ionic strength. The procedure of trimming the REFET is, however, not very practical in a differential ISFET/REFET measurement set-up, in which other requirements should also be fulfilled, such as a zero offset at, for instance, $\mathrm{pH}=\mathbf{7}$ or an isothermal bias point.

\section{Non-blocking REFETs}

\section{Working mechanism}

The electrical problems mentioned in the previous Section can in principle be prevented by the use of a non-blocking (or unblocked) layer, or in other words by the use of a conventional membrane as also applied for CHEMFETs, for instance, a potassium-sensitive FET. If the ionconducting properties of such a membrane are sufficient to pass the electrical voltage of the aqueous solution to the original gate of the underlying ISFET, the transconductance of the coated ISFET remains equal to the original transconductance. If, furthermore, an ion-exchange process is the potential-determining mechanism of the membrane/solution interface, charging of the corresponding double layer by an externally applied voltage is decreased drastically and thus so is the influence of the ionic strength. Of course, the membrane should also be insensitive to variations in specific ion concentrations and the interface between the membrane and the gate oxide should be thermodynamically well defined. The development of such membrane-coated ISFETs will be described below.

\section{Suitable membrane materials}

Besides the requirement of conductivity and ion insensitivity, an important aspect of polymeric layer-type REFETs is the polymer attachment to the gate oxide.

In the case of ion-blocked, plasma-polymerized layers, as described in one of the previous Sections, a stable attachment may be guaranteed by the presence of highly reactive and polymerizable radical monomer molecules, produced in the plasma. Conversely, ion unblocked, hydrophobic membranes are seldom well bound to the surface if no special precautions are taken. Keeping these considerations in mind, materials that offer the possibility of covalent chemical attachment to the surface have to be selected and investigated. In addition, it would be advantageous if the polymer layers could be deposited by photolithographic methods. These requirements have led to the investigation of the following materials. 
Vernis pour l'Electronique (VE)

VE-REFETs were prepared by spray-coating $\mathrm{SiO}_{2}$ or $\mathrm{Ta}_{2} \mathrm{O}_{5}$ ISFETs with a commercially available plastic insulator spray (Siceront, France). In this way, layer thicknesses of $1-3 \mu \mathrm{m}$ were obtained, as measured by interferometry.

Bis-acrylate (BA)

Bis-acryl 101 (AKZO) was applied by spin-coating as a solution in tetrahydrofuran (THF) with $4 \%$ (wt.) dimethoxyphenylacetophenone as a photoinitiator on the surface of $\mathrm{SiO}_{2}$ ISFETs, which were previously silylated with trimethoxysilylpropyl methacrylate (TSPM). Subsequently the (monomeric) layer was photopolymerized by irradiation for $1 \mathrm{~s}$ with the ultraviolet light of a mask aligner (Karl Süss). This photopolymerization had to be carried out under strictly oxygen-free conditions in order to avoid quenching of the polymerization. With this technique, layer thicknesses of $0.4-1.0 \mu \mathrm{m}$ could be achieved (interferometry). These layers were used in the experiments from which the results have been given in Fig. 3. For the sake of developing an unblocked membrane, $0.01 \%$ (wt.) potassium tetraphenylborate $\left(\mathrm{KBPh}_{4}\right)$ was added to the bis-acrylate in order to investigate the effect of induced ion conductivity.

Polybutadiene (PBD)

PBD layers were applied to silylated $\mathrm{SiO}_{2}$ ISFET surfaces (see above) by solvent casting. For this, a solution of $10-20 \%$ (wt.\%) PBD in THF with $4 \%$ (wt.) benzophenone as the photoinitiator was used. The layers were polymerized and attached to the surface by irradiation with ultraviolet light for $30 \mathrm{~min}$, and were subsequently cured at $80^{\circ} \mathrm{C}$ for $18 \mathrm{~h}$. According to interferometry, typical layer thicknesses of $7-15 \mu \mathrm{m}$ were obtained.

Acrylate ( $A C$ )

Various mixtures of ACE* and Epocryl DRH 370* (such mixtures are denoted as acrylates or AC) with $4 \%$ benzophenone as the photoinitiator were used to form polyacrylate layers by photopolymerization. It appeared that, for valuable measurements with REFETs fabricated with acrylate, a poly(hydroxyethyl methacrylate) (p-HEMA) layer had to be interposed between the $\mathrm{SiO}_{2}$ surface and the polymer. The function of p-HEMA and the method of application of such layers will be discussed in another paper. The polyacrylate layers were applied on the p-HEMA ISFET surfaces by solvent casting. The polymerization of the acrylates was carried out by irradiation (ultraviolet light) for 2 - $4 \mathrm{~min}$. Layer thicknesses varied from 2 to $5 \mu \mathrm{m}$ (interferometry).

*ACE and Epocryl are mono- and bifunctional acrylate-type monomers, kindly supplied by Koninklijke Shell Laboratorium, Amsterdam. 
From all membrane materials mentioned above, REFETs were constructed and approved by physical inspection as serious candidates for further investigation.

The detailed chemical aspects of the modification process are not the subject of this paper, but the experience is that by means of covalently anchoring of acrylate-type polymers on presilylated $\mathrm{SiO}_{2}$, a bond that will last at least three months can be obtained under conditions of continuous immersion in aqueous electrolyte solutions. An important aspect is, of course, the electrical behaviour, besides the chemical responsivity. Both aspects will be considered in some detail in the following Sections.

\section{Electrical relaxation time}

The electrical behaviour of polymeric layers can best be characterized by their electrical relaxation time. In ion-unblocking REFET structures, ion exchange takes place between the solution and the polymeric layer. With ion-blocking structures, this process occurs to a negligible extent. An essential consequence is that in unblocked structures, with a thermodynamic equilibrium between ions in solution and in the membrane, the measured potential is a membrane potential. In a blocked structure, the measured potential is a surface potential, formed by surface complexation reactions. In order to discriminate between the two types of structures, a criterion has to be formulated. The most crucial difference between blocked and unblocked layers in contact with an aqueous solution is the possibility of ion exchange, which requires a certain ion concentration and ion mobility in the membrane. Therefore, the electrical relaxation time $\tau$ is a good criterion to discriminate between the two mechanisms. The relaxation time is a measure for the time needed to redistribute the charge and establish potential profiles in the polymer after application of an electrical field. The parameter $\tau$ is given by:

$\tau=\rho \epsilon=R_{\mathrm{pol}} C_{\mathrm{pol}}$

with $\rho=$ resistivity of the polymer, $\epsilon=\epsilon_{\mathrm{r}} \epsilon_{0}=$ permittivity of the polymer, $R_{\mathrm{pol}}=$ polymer bulk resistance and $C_{\mathrm{poi}}=$ polymer bulk capacitance, and indicates the time needed for an electrically-induced potential difference across the polymer to decrease to 1 /e times its original value.

Considering the whole range of possible $\tau$ values, there are two regions that are of interest for REFET-applicable polymers. The first region is that of very large $\tau$ values (quasi-blocked layers). If a certain potential difference is applied across the membrane, extremely large values of $\tau$ ensure that the voltage decay rate (which is experienced as drift in a REFET measurement) remains within reasonable limits (for a drift rate of $1 \mathrm{mV} / \mathrm{h}$ and an applied voltage of $1 \mathrm{~V}$, a value of $\tau>300 \mathrm{~h}$ can be estimated). In the case of unblocked layers, the relaxation time $\tau$ has to be small enough to follow fast changes in potential. In this paper, a value of $\tau=10 \mathrm{~s}$ will be used as a criterion to distinguish between the two types of structures. In the following Section, several electrical methods will be described that can be used to mea- 
sure the electrical relaxation time. Furthermore, SIMS measurements are used to relate these experimental results to the degree of ion penetration in the polymer after contact with electrolyte solutions.

\section{Measurement techniques for electrical characterization}

If a polymeric layer is represented by a parallel $\left(R_{\mathrm{pol}} / / C_{\mathrm{pol}}\right)$ network in series with the gate oxide, the electrical relaxation time $\tau$ corresponds to the product $R_{\text {pol }} C_{\text {pol. }}$. The value of $\tau$ can be determined from the relation between the electrical transistor transconductance $g_{m}$ and the frequency of the sinusoidal signal that is applied to the transistor. The transconductance $g_{m}$ can be determined directly by measuring $\partial I_{d} / \partial V_{g s}$ with an open-loop measuring system.

However, since the application of ISFETs is usually carried out with a source and drain follower (closed-loop) amplifier, it is worthwhile to investigate whether $\tau$ can also be determined in this arrangement. In the source and drain follower the drain current $I_{d}$ is kept constant by means of feedback, which implies that $g_{m}$ cannot be measured in this way. Therefore, two other methods have been investigated in addition to the $g_{m}$ measurements, in order to determine the electrical relaxation time $\tau$ under constant drain current conditions.

The first method is bulk modulation. With this technique the (frequency-dependent) change in $V_{\mathrm{gs}}$, needed to keep $I_{\mathrm{d}}$ constant if the sourcebulk voltage $V_{\mathrm{sb}}$ is varied, supplies the value of $\tau$. The second method is the light-pulse response. Now, the electrical relaxation time can be deduced from the shape of the $V_{\mathrm{gs}}$ versus time curve after the application of a light pulse.

Transconductance measurements (TM)

The transconductance of an unmodified ISFET is defind as:

$g_{\mathrm{m}}=\frac{\partial I_{\mathrm{d}}}{\partial V_{\mathrm{gs}}}=\frac{i_{\mathrm{d}}}{v_{\mathrm{gs}}}$

with $i_{\mathrm{d}}=$ small-signal drain current and $v_{\mathrm{gs}}=$ small-signal gate-source voltage. The $g_{m}$ was measured with the open-loop arrangement depicted in Figure 4. The small-signal output voltage $v_{\text {out }}$ is given by

$v_{\text {out }}=-R i_{\mathrm{d}}=-R g_{\mathrm{m}} v_{\mathrm{gs}}$

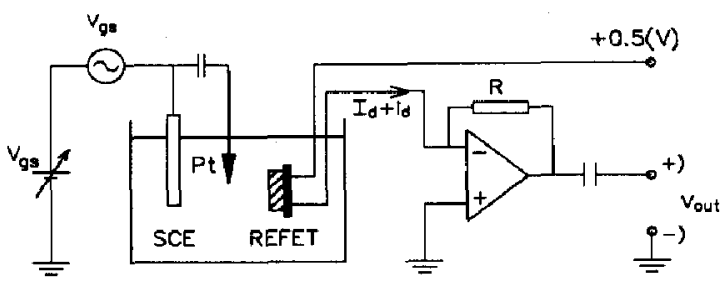

Fig. 4. Open-loop measuring system for transconductance determination. 
If a polymeric layer is placed on top of the gate insulator, the voltage $v_{\mathrm{gs}}$ is divided over the polymer and the oxide. In this case, the transfer function $H(j \omega)$ has to be introduced in eqn. (3) as a multiplication factor for $v_{\mathrm{gs}}$ :

$H(\mathrm{j} \omega)=\frac{1+\mathrm{j} \omega R_{\mathrm{pol}} C_{\mathrm{pol}}}{1+\mathrm{j} \omega R_{\mathrm{pol}}\left(C_{\mathrm{pol}}+C_{\mathrm{ox}}\right)}$

with $C_{\mathrm{pol}}=$ capacitance of the polymeric layer $(F), R_{\mathrm{pol}}=$ resistance of the polymeric layer $(\Omega), C_{\text {ox }}=$ oxide capacitance $(\mathrm{F})$ and $\omega=$ angular frequency $\left(\mathrm{rad} \mathrm{s}^{-1}\right)$. This means that $g_{\mathrm{m}}\left(=-v_{\text {out }} /\left(\boldsymbol{R H}(\mathrm{j} \omega) v_{\mathrm{gs}}\right)\right.$ becomes frequency dependent. In Fig. 5 the dependence of $|H(j \omega)|$ on frequency is presented, with $\tau_{1}=R_{\mathrm{pol}}\left(C_{\mathrm{pol}}+C_{\mathrm{ox}}\right)$ and $\tau_{2}=R_{\mathrm{pol}} C_{\mathrm{pol}}$. The time constant $\tau_{2}$ is the electrical relaxation time of the polymer.

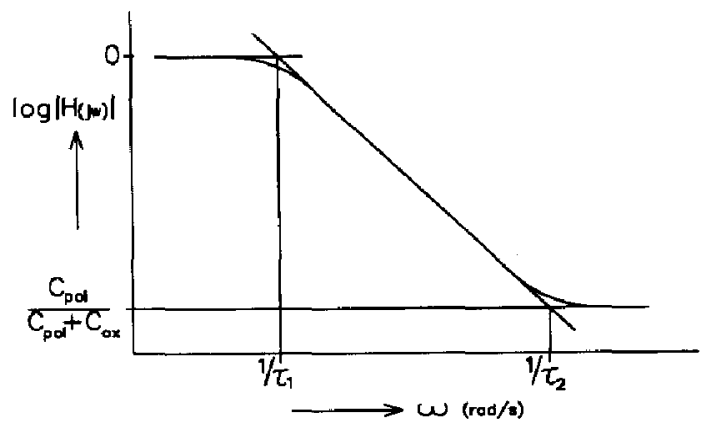

Fig. 5. Schematic representation of transfer function $H(j \omega)$.

\section{Bulk modulation (BM)}

From semiconductor theory [12] it is well known that for small variations of the source-bulk voltage $V_{\mathrm{sb}}$, the threshold voltage $V_{\mathrm{T}}$ changes according to:

$V_{\mathrm{T}}=V_{\mathrm{TO}}+\left(n_{0}-1\right) V_{\mathrm{sb}}$

where

$V_{\mathrm{T} 0}=V_{\mathrm{FB}}+2 \phi_{\mathrm{b}}+K \sqrt{2 \phi_{\mathrm{b}}}$

$V_{\mathrm{FB}}=$ flat-band voltage

$n_{0}=1+K /\left(2 \sqrt{2 \phi_{\mathrm{b}}}\right)$

$=\sqrt{2 q \epsilon_{\mathrm{S} 1} N_{\mathrm{b}}} / C_{\mathrm{ox}}$

$\phi_{\mathrm{b}}=\left(\frac{k T}{q}\right) \ln \frac{N_{\mathrm{b}}}{N_{\mathrm{i}}}$

with $k T / q=0.025$ (V) at $T=300 \mathrm{~K}, N_{\mathrm{b}}=$ concentration of impurity (doping) charge carriers $\left(\mathrm{m}^{-3}\right), N_{\mathrm{i}}=$ intrinsic carrier concentration $\left(\mathrm{m}^{-3}\right)$ and $\epsilon_{\mathrm{Si}}=$ permittivity of silicon $\left(\mathrm{F} \mathrm{m}^{-1}\right)$. 
If the appropriate values of the various parameters are substituted in the formulae, the dependence of the threshold voltage on source-bulk voltage variations is given by:

$$
\Delta V_{\mathrm{T}} / \Delta V_{\mathrm{sb}}=\mathbf{0 . 3 3}
$$

This means that for an ISFET in constant drain-current mode, the application of a voltage pulse between source and bulk $\left(\Delta V_{\mathrm{sb}}\right)$ results in a compensating pulse of the gate voltage $\Delta V_{\mathrm{zs}}=0.33 \Delta V_{\mathrm{sb}}$, which is divided over the polymer layer and the gate oxide. The voltage applied across the polymer shows an exponential decay, with a time constant determined by the relaxation time $\tau=R_{\mathrm{pol}} C_{\mathrm{pol}}$ of the polymer. The source and drain follower circuit regulates $V_{\mathrm{gs}}$ in order to compensate this effect such that $I_{\mathrm{d}}$ remains constant, and thus a corresponding exponential behaviour of $V_{\mathrm{ks}}$ is experienced, as shown in Fig. 6.

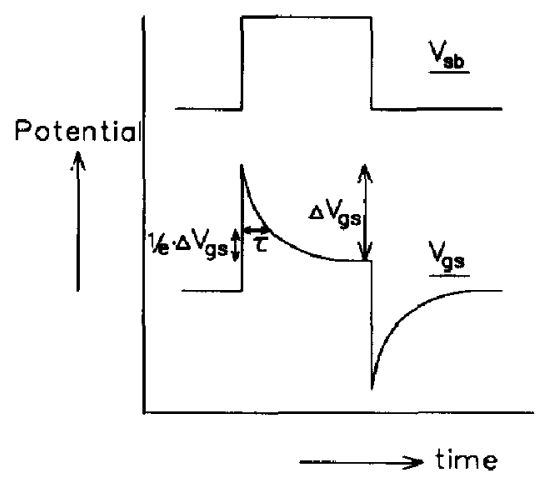

Fig. 6. Schematic representation of $V_{\mathrm{Cg}}$ response with respect to a block-wise change of $V_{\text {sb. }}$

The electrical relaxation time $\tau$ is determined from the $V_{\mathrm{gs}}$ versus time curves by measuring the time needed for $\Delta V_{\mathrm{gs}}$ to decay to $1 / \mathrm{e}$ times its initial value (see Fig. 6). The frequency range of this method is limited by the characteristics of the source and drain follower circuit.

Light pulse response (LPR)

In the operation of ISFETs, there are two effects responsible for the influence of illumination on the $I_{\mathrm{d}}-V_{\mathrm{ds}}$ characteristics: drain-bulk diode photocurrents, and threshold voltage shift due to photogenerated charge carriers in the inversion layer [13]. For both effects, the source and drain follower arrangement keeps $I_{d}$ constant by adapting $V_{\mathrm{gs}}$, which means that the bias point of the ISFET will change. Therefore a light pulse will result in a $\Delta V_{\mathrm{ks}}$, again divided over the polymer and the oxide; the part that is applied across the polymer exhibits the same exponential decay as in the case of bulk modulation. 
Results and discussion

The results of transconductance measurements carried out with Vernis pour l'electronique (VE), poly(butadiene) (PBD) and bis-acrylate (BA), without and with added $\mathrm{KBPh}_{4}$, are given in Fig. 7, and indicate that VE and PBD behave like ion-unblocked polymers $(\tau \ll 10 \mathrm{~s})$, whereas pure BA can be considered to be an ion-blocked polymer $(\tau \gg 10 \mathrm{~s})$. This is the layer already experienced in Figs. 2 and 3. If, however, $\mathrm{KBPh}_{4}$ is added, the results of Fig. 7 indicate that the characteristic behaviour shifts to an ion-unblocked type.

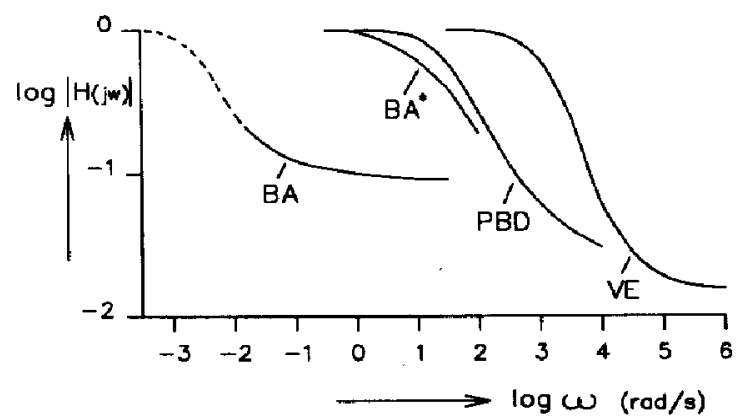

Fig. 7. REFET transfer function $H(j \omega)$ for VE, PBD and BA REFETs (BA* = BA after addition of $0.1 \%$ (wt.) $\mathrm{KBPh}_{4}$ ).

Bulk modulation and light pulse measurements carried out with ACREFETs (the polymer on top of the conducting hydrogel poly-HEMA, which does not influence the relaxation time) resulted in relaxation times of $\tau=0.35 \mathrm{~s}$ and $\tau=0.40 \mathrm{~s}$ respectively for the methods mentioned. This result shows that both methods are suitable to give an easy and rapid estimation of the electrical relaxation time of the polymer layer.

PBD was further investigated with SIMS. Cationic concentration profiles recorded before and after immersion of the polymer in an aqueous $10^{-3} \mathrm{M} \mathrm{KCl}$ solution are displayed in Figs. 8(a) and (b). From these profiles it is concluded that the short relaxation time of PBD results from a substantial uptake of ions from the sample solution.

The pH sensitivity of the fabricated REFETs, one of their most important properties, was measured with a source and drain follower circuit.

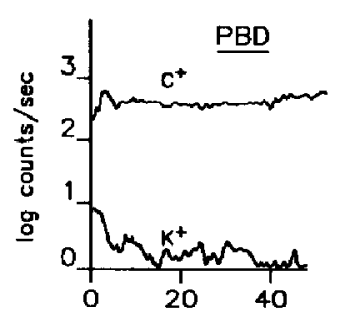

(a) sputt. time (min.)

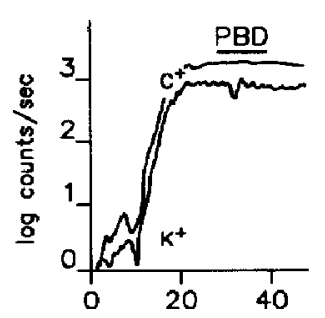

(b) sputt. time (min.)

Fig. 8. Cationic SIMS profiles of PBD-REFETs, (a) before and (b) after immersion in $10^{-3} \mathrm{M} \mathrm{KCl}$ for $2 \mathrm{~h}$. 


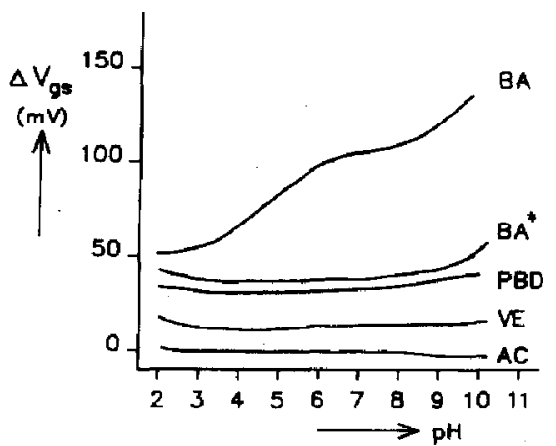

Fig. 9. pH response of AC-, VE-, PBD-, and BA-REFETs. (BA* = BA with added methacrylic acid.)

The results are given in Fig. 9. Each of the REFETs exhibited a very small $\mathrm{pH}$ response, except for the BA-REFET, as already mentioned. As already suggested in a previous Section, this observed $\mathrm{pH}$ sensitivity might result from the presence of surface acrylic acid groups and therefore one would expect that the $\mathrm{pH}$ sensitivity would increase upon addition of acid. However, after adding an extra amount of methacrylic acid to the BA monomer, a polymer was formed that exhibited a much lower $\mathrm{pH}$ response (BA* in Fig. 9). The reason for this effect is probably that the properties of the polymer are changed in such a way that ion exchange instead of surface complexation has become the potential determining process, as in the case of the addition of $\mathrm{KBPh}_{4}$, which changed the relaxation time to much lower values.

Although REFETs made from the other polymers (VE, PBD, AC) showed a very low $\mathrm{pH}$ sensitivity when a relatively fast $\mathrm{pH}$ scan was applied (approx. $10 \mathrm{~min}$ ), much slower scans exhibited a large hysteresis effect, which is observed as pseudo-drift for both VE and PBD devices. It may be concluded that $\mathrm{H}^{+}$and $\mathrm{OH}^{-}$diffusion do take place to a considerable extent in these devices if a certain $\mathrm{pH}$ is 'sensed' for a long period of time. This effect makes these REFETs useful only under restricted conditions. The acrylate REFETs did not exhibit this pseudo-drift behaviour, as shown in Fig. 10.

\section{Conclusions}

The results of all experiments described above are summarized in Table 1 , from which the final conclusion may be drawn that from all the original candidates, the acrylate REFET is the most interesting type to be investigated in more detail.

\section{Acrylate type REFETs}

\section{Permselectivity}

From the preceding results, the conclusion seems to be that the nonblocking AC-REFET fulfills all the requirements for a well-functioning 


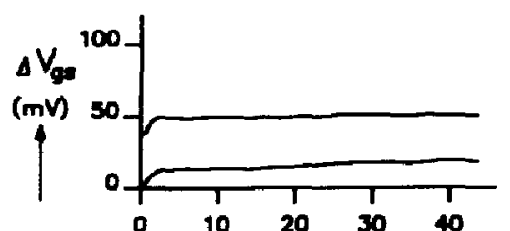

(a)

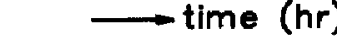

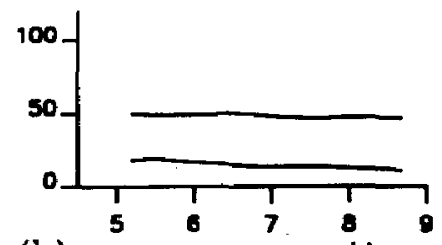

(b)

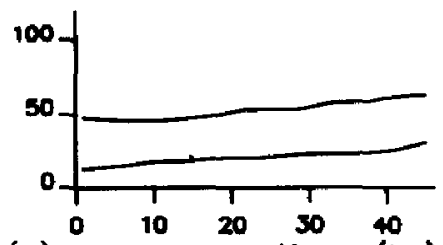

(c)

time (hr)

Fig. 10. pH response (b) and time courses of 2 AC-REFETs, (a) before and (c) after the pH scan.

TABLE 1

Summary of experimental results

\begin{tabular}{llllll}
\hline & $\begin{array}{l}\text { Relaxation } \\
\text { time } \tau(\mathrm{s})\end{array}$ & $\begin{array}{l}\text { Operational } \\
\text { mechanism }\end{array}$ & $\begin{array}{l}\mathrm{pH} \text { sensitivity } \\
(\mathrm{mV} / \mathrm{pH})\end{array}$ & $\begin{array}{l}\text { Pseudo drift } \\
(\mathrm{mV} / \mathrm{h})\end{array}$ & Valuation \\
\hline $\mathrm{VE}$ & $<10$ & - & $<5$ & $>10$ & - \\
$\mathrm{BA}$ & $>10$ & no ion exchange & 10 & - & - \\
$\mathrm{BA}+\mathrm{KBPh}_{4}$ & $<10$ & ion exchange & $<5$ & - & \pm \\
$\mathrm{PBD}$ & $<10$ & ion exchange & $<5$ & $>10$ & - \\
$\mathrm{AC}$ & $<10$ & - & $<2$ & $<0.25$ & + \\
\hline
\end{tabular}

REFET: it has (a) no (fast or slow) pH response; (b) chemical attachment to the gate oxide; (c) transconductance equal to that of the ISFET for frequencies of interest. Yet, despite these satisfying properties, there is one disadvantage exhibited by all non-blocking REFET polymers (except VE): cation permselectivity. This term indicates that the membranes are merely permeable for cations, which is a property of many polymeric membranes. This phenomenon has been subject to many speculations in the literature of poly(vinyl chloride) membrane electrodes [14] and may have two different origins.

The first is the much lower mobility of anions in the polymer if compared with cations. This difference leads to the formation of a membrane diffusion potential. The second source of permselectivity is the presence of immobile anionic sites in the membrane. The fixation of the concentration of exchangeable cations by these sites leads to the formation of a boundary potential.

It is obvious that in order to influence this behaviour, the membrane composition must be changed. Addition of large, lipophilic cations, a method that has been employed to construct anion ion-exchange membranes [15], might affect both mechanisms. The concentration of mobile cations can be decreased in this way by competitive exchange with immobile lipophilic cations. Therefore a change of permselective behaviour is to be expected.

With respect to the AC-REFETs, various amounts of di-dodecyldimethylammonium bromide (DDMAB) were added to the acrylate polymer, and the cation response of the REFETs made with this material was mea- 

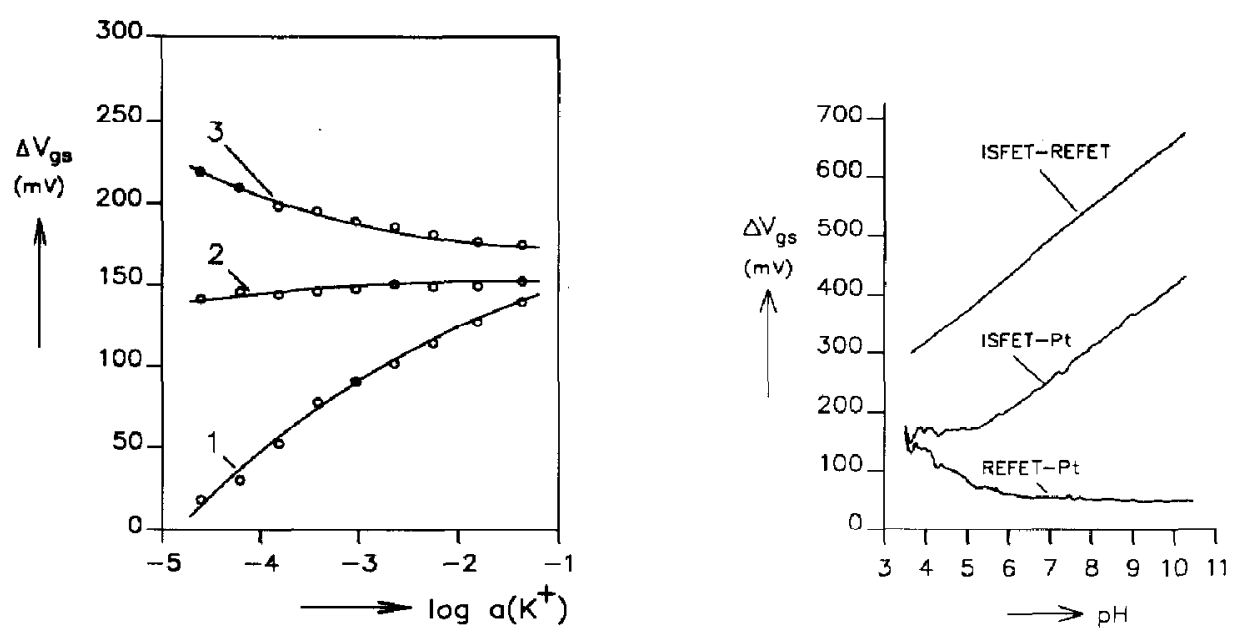

Fig. 11. Potassium response of AC-REFETs with various amounts of added DDMAB. (1) $[\mathrm{DDMAB}]=0 \mathrm{~mol} / \mathrm{g} ;$ (2) $[\mathrm{DDMAB}]=2 \times 10^{-5} \mathrm{~mol} / \mathrm{g} ;$ (3) $[\mathrm{DDMAB}]=9 \times 10^{-5}$ $\mathrm{mol} / \mathrm{g}$.

Fig. 12. Response to pH of ISFET/Pt, AC-REFET/Pt and ISFET/AC-REFET system.

sured. The results with respect to $\mathrm{K}^{+}$ion response are given in Fig. 11 . From the responses, we conclude that with the proper amounts of ionic components present in the polymer, the permselective concentration response can be almost completely eliminated (curve 2). In other words, the AC-REFETs can be trimmed with respect to insensitivity to concentration variations.

\section{Differential measurements}

As already mentioned in the Introduction, in an ISFET/REFET differential measurement, the REFET is used to compensate and eliminate the unstable potential of the quasi-reference electrode (QRE) (see Fig. 1). An important parameter to indicate the suppression of varying reference electrode potentials is the common mode rejection ratio (CMMR). This factor, which is normally frequency dependent, is in this application mainly of interest for very low frequencies and d.c. signals. Hence, d.c. variations of $\pm 1.0 \mathrm{~V}$ were applied to a saturated calomel electrode (SCE) to investigate the CMMR (a SCE was used rather than a QRE to ensure the application of the proper potential). It was found that the ISFET-AC-REFET-SCE system shows a CMMR $>1000$. This relatively high value is ascribed to the equality of the electrical transconductances of the ISFET and REFET, although the REFET has a duplex poly-HEMA/acrylate membrane of $\cong 5 \mu \mathrm{m}$. It may therefore be concluded that, under normal circumstances (QRE-potential variations $<100 \mathrm{mV}$ ), fluctuations in the potential of the QRE will have no disturbing influence on the measurement.

The results of an AC-REFET/ $\mathrm{Al}_{2} \mathrm{O}_{3}$-ISFET/Pt couple are given in Fig. 12, together with the measurements of the separate ISFET and REFET 
respectively with respect to the Pt-QRE. These results show clearly how the unstable QRE potential is measured by the ISFET as well as the REFET and that this potential is eliminated in the actual differential ISFET/REFET measurement.

An additional advantage of the differential measurement is the reduction of light sensitivity with respect to a single ISFET. This effect was investigated by changing the radiation intensity from ambient light to illumination with a tungsten lamp at $30 \mathrm{~cm}$ distance. A reduction factor $>20$ was measured in relation to a single ISFET device.

Although acrylate membranes already form a better barrier to $\mathrm{CO}_{2}$ than PVC membranes, the influence of $\mathrm{CO}_{2}$ as described by Fogt et al. [16] is completely eliminated due to the application of an intermediate p-HEMA layer, which contains a Merck buffer $\mathrm{pH}=4$.

\section{Conclusions}

It was shown that it is possible to fabricate reference FETs by chemical attachment of ion-conducting polymeric membranes to a pretreated ISFET gate surface, without decreasing the transconductance of the original ISFET. With AC-REFETs the pH sensitivity is almost completely eliminated $(<1$ $\mathrm{mV} / \mathrm{pH}$ ), and no slow $\mathrm{pH}$ response is observed. The intrinsic polymer permselectivity can be significantly reduced by the addition of a balanced amount of immobile cationic sites to the polymer. Differential measurements with an ISFET/REFET pair show that fluctuations in the QRE potentials are sufficiently eliminated and that an additional positive effect of the differential system is the suppression of light sensitivity.

\section{Acknowledgements}

The considerations given in this paper have been stimulated by the cooperation between the Biosensor Research Group, headed by the first author, and the Laboratory of Organic Chemistry, headed by the last author. The actual experimental results reported here are part of the thesis of the second author, while the help of the other authors, all from the Laboratory of Organic Chemistry, was indispensable. The financial support from the Centre of Microelectronics is greatly appreciated, especially with respect to the stimulation concerning the actual application of the FET chemical sensors.

\section{References}

1 P. Bergveld and A. Sibbald, Analytical and Biomedical Applications of ISFETs, Elsevier, Amsterdam, 1988.

2 R. L. Smith and D. C. Scott, IEEE Trans. Biomed. Eng., BME-33 (1986) 83 - 90. 
3 T. Matsuo and M. Esashi, 153rd Meet. Electrochem. Soc. Ext. Abstr., 78-1 (1978) $202-203$.

4 R. P. Buck, Biosensors based on reversible reactions at blocked and unblocked electrodes, J. Chem. Soc. Faraday Trans. 1, 82 (1986) $1169-1178$.

5 M. Fujihira, M. Fukui and T. Osa, Preparation of $\mathrm{pH}$-insensitive parylene gate for chemical modification, J. Electroanal. Chem., 106 (1980) 413 - 418.

6 L. Bousse, N. F. de Rooij and P. Bergveld, Operation of chemically sensitive fieldeffect sensors as a function of the insulator-electrolyte interface, IEEE Trans Electron Devices, $E D-30$ (1983) 1263 - 1270.

7 A. van den Berg, P. Bergveld, D. N. Reinhoudt and E. J. R. Sudhölter, Sensitivity control of ISFETs by chemical surface modification, Sensors and Actuators, 8 (1985) $129-148$.

8 H. Nakajima, M. Esashi and T. Matsuo, The cation concentration response of polymer gate ISFET, J. Electrochem. Soc., 129 (1982) 141 - 143.

9 S. Tahara, M. Yoshii and S. Oka, Electrochemical reference electrode for the ionselective field effect transistor, Chem. Lett., (1982) $307-310$.

10 A. van den Berg, P. D. van der Wal, D. Ptasinski, E. J. R. Sudhölter, D. N. Reinhoudt and $P$. Bergveld, Preparation and characterization of REFETs prepared by chemical surface modification of ISFETs, Proc. 2nd Int. Meet. Chem. Sensors, Bordeaux, France, July 7 - 10, 1986 , pp. $419 \cdot 422$.

11 T. Matsuo and H. Nakajima, Characteristics of reference electrodes using a polymer gate ISFET, Sensors and Actuators, 5 (1984) 293 - 305.

12 S. M. Sze, Physics of Semiconductor Devices, Wiley, New York, 2nd edn., 1981, Ch. 8.

$13 \mathrm{~W}$. Wlodarski, P. Bergveld and J. A. Voorthuyzen, Threshold voltage variations in n-channel MOS transistors and MOSFET-based sensors due to optical radiation, Sensors and Actuators, 9 (1986) $313-321$.

14 A. van den Berg, P. D. van der Wal, M. Skowronska-Ptasinska, E. J. R. Sudhölter, D. N. Reinhoudt and P. Bergveld, Nature of ionic sites in plasticized poly(vinyl chloride) membranes, Anal. Chem., 59 (1987) 2827 - 2929.

$15 \mathrm{X}$. Wei-zhen, S. Jian-lin and $\mathrm{H}$. Jin-hua, The preparations and properties of PVC salicylate ion-selective electrode, Proc. 2nd Int. Meet. Chem. Sensors, Bordeaux, France, July 7 - 10, 1986 , pp. 491 - 494 .

16 E. J. Fogt and M. E. Meyerhoff, Response of ion selective field effect transistors to carbon dioxide and organic acids, Anal. Chem., 57 (1985) $1995 \cdot 1998$.

\section{Biographies}

Piet Bergueld was born in Oosterwolde, the Netherlands, on January 26, 1940. He received the M.S. degree in electrical engineering from the University of Eindhoven, the Netherlands, in 1965 and the Ph.D. degree from the University of Twente, the Netherlands, in 1973. The subject of his dissertation was the development of ISFETs and related devices, since then also investigated by many international research groups of Universities as well as industry.

Since 1965 he has been a member of the Biomedical Engineering Division of the faculty of Electrical Engineering (University of Twente) and was in 1984 appointed as full professor in Biosensor Technology. He is the chairman of the research unit Sensors and Actuators.

His research subjects still concern the further development of ISFETs and biosensors based on ISFET technology. 
David $N$. Reinhoudt was born in Wolfaartsdijk, the Netherlands, on September 18, 1942. He received his M.S. degree in chemical engineering (organic chemistry) from the Delft University of Technology in 1966, and his Ph.D. degree from the same institute in 1969 (with honours). From 1970 until 1975 he was research chemist at the Koninklijke/Shell Laboratories in Amsterdam (organic chemistry section). In 1975 he was appointed as professor in organic chemistry at the University of Twente.

Maria Skowronska-Ptasinska was born in Radom, Poland, on January 4, 1947. She received her M.S. degree in chemical engineering from the Warsaw Technical University in 1969, and her Ph.D. degree in chemistry from the same University in 1975. From 1970 until 1980 she worked at the Department of Chemistry, Warsaw Technical University (organometallic chemistry). In $1980-1981$ she was appointed as associate research assistant at the Department of Chemistry, University College, London, U.K. From 1983 until 1988 she has worked at Twente University on organic synthesis of macrocyclic receptors.

Ernst J. R. Sudhölter was born in Hengelo, the Netherlands, on November 23, 1953. He received his M.S. degree in organic chemistry from the University of Groningen in 1977, and his Ph.D. degree (cum laude) from the same University in 1981 . From 1981 until 1984 he was research chemist at the Koninklijke/Shell Laboratories in Amsterdam in the fundamental and process development departments. Since 1984 he has worked at the University of Twente on physical organic chemistry of surfaces and membranes.

Albert van den Berg was born in Zaandam, the Netherlands, on September 20, 1957. He received his M.S. degree in (technical) physics in 1983 from the University of Twente. Since then he has been working on the ion sensor research project, which is being carried out in cooperation between the Biosensor Research Group and the Department of Organic Chemistry of the University of Twente and received his Ph.D. degree in January 1988. Since then he has been working at CSEM, Neuchâtel, Switzerland.

Peter D. van der Wal was born in Gaasterland, the Netherlands, on May 21,1959 . He received his M.S. degree in chemical engineering in 1985 from the University of Twente. Since then he has been working as a research chemist at the University of Twente on the ion sensor research project. 